DOI: https://doi.org/10.24867/02IH07Boberic

\title{
JEDNO OD REŠENJA ZA PARTICIONISANJE CENTRALNE PROCESORSKE JEDINICE SA VIŠE JEZGARA
}

\section{ONE SOLUTION FOR PARTITIONING MULTICORE CENTRAL PROCESSING UNIT}

\author{
Milan Boberić, Fakultet tehničkih nauka, Novi Sad
}

\section{Oblast -MEHATRONIKA}

Kratak sadržaj - U ovom radu predstavljena je implementacija i optimizacija sistema za particionisanje centralne procesorske jedinice sa više jezgara. Sistem se sastoji iz UltraZed-EG ploče na kojoj je implementiran Xen Hypervisor kao sistem za particionisanje. Xen Hypervisor je virtuelni sloj koji koristi postojeći operativni sistem (host) koji mu omogućava pristup memoriji, prekidima $i$ drugim resursima $i$ omogućava kreiranje virtuelnih jezgara koja se po instrukcijama korisnika mogu raspoređivati na fizička jezgra. Dat je akcenat na real-time particionisanje $i$ optimizaciju Xen Hypervisora u cilju poboljšanja performansi.

Ključne reči: Particionisanje centralne procesorske jedinice, Xen Hypervisor, virtuelizacija, UltraZed-EG

\begin{abstract}
This master thesis presents the implementation and optimization of a system for partitioning a multicore central processing unit. The system contains an UltraZed-EG board on which Xen Hypervisor is implemented as the partitioning system. Xen Hypervisor is a virtual layer which uses the existing operative system (host) to access memory, interrupts, and other resources and allows creation of virtual cores which can be scheduled across physical cores. Accent is given on real-time partitioning and Xen Hypervisor's optimization in order to improve it's performance.
\end{abstract}

Keywords: Central processing unit partitioning, Xen Hypervisor, virtualization, UltraZed-EG

\section{UVOD}

Particionisanje centralne procesorske jedinice sa više jezgara znači podela grupe jezgara ili pojedinačnih jezgra za specijalnu upotrebu jedne ili više aplikacija. Svaka sekcija se ponaša kao zaseban sistem sa promenljivim stepenom fleksibilnosti raspodele. Real-time particionisanje sistema opisuje izloženost hardvera i softvera real-time ograničenjima, na primer od nekog događaja do reakcije sistema na taj događaj, real-time programi moraju garantovati reakciju sistema $u$ određenom vremenskom intervalu.

Jedan od sistema za particionisanje jeste Xen Hypervisor. U ovom radu je opisana implementacija Xen Hypervisora sistema za particionisanje kao i poređenje performansi sistema sa i bez Xen Hypervisor-a na ploči UltraZed-EG kao i na ploči ZedBoard.

\section{NAPOMENA:}

Ovaj rad proistekao je iz master rada čiji mentor je bio dr Vladimir Rajs, docent.
Pored drugih hypervisor-a kao što su: VMware, ESXi, Hyper-V, KVM, itd. Xen Hypervisor ima cenu, performance i sigurnost da parira najboljim na svetu. Ima deset godina iskustva u radu sa najvećim cloud-ovima na svetu. Siguran je, stabilan i proveren izbor za virtuelizaciju koji koriste giganti u industriji kao što su: Amazon, Rackspace, Verizon, itd.

\section{HARDVER}

U okviru projekta koristi se UltraZed-EG ploča sa nosačom kartice. UltraZed-EG SOM (system on module) je visoko fleksibilan, robustan system-on-module baziran na Xilix Zynq UltraScale+MPSoC. UltraZed-EG omogućava lak pristup ka $180 \mathrm{I} / \mathrm{O}$ pinova, 26 PS MIO pinova i četiri $P S$ GTR transivera velike brzine sa četiri GTR referentna klok ulaza koja imaju tri I/O konektora na poleđini modula. Takođe sadži Quad-core Cortex A53, Dual-core Cortex-R5 real-time procesor i ARM MALI 400MP GPU gde su 4 jezgra Cortex A53 procesora na raspolaganju za virtuelizaciju. $\mathrm{Na}$ slici 1 je prikazan izgled ploče.

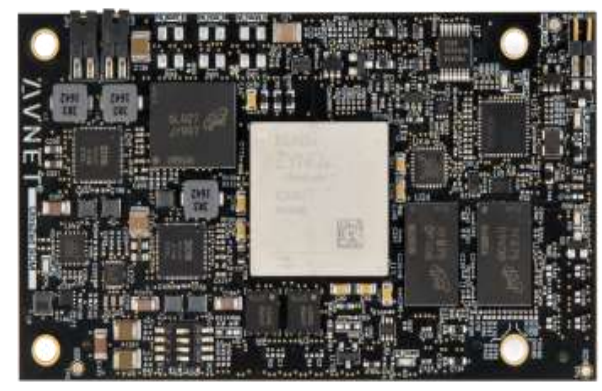

\section{Slika 1 - Izgled UltraZed-EG ploče [1]}

\section{XEN HYPERVISOR U LINUX OPERATIVNOM SISTEMU}

Xen je virtuelni sloj kao što je prikazano na slici 2. Na osnovu virtuelnih mašina $(V M)$ i podešavanja Xen Hypervisor-a, VM-e se raspoređuju po fizičkim jezgrima $(p C P U)$ i imaju pristup određenim delovima fizičkog hardvera u zavisnosti od passthrough-a.

U ovom slučaju host tj. dom0 je PetaLinux, Xen guests su totalno izolovani od hardvera i nemaju pristup ni hardveru niti I/O funkcijama i nazivaju se domU. Xen Hypervisor neće funkcionisati bez dom0 koji je prva virtuelna mašina koju sistem pokrene. 


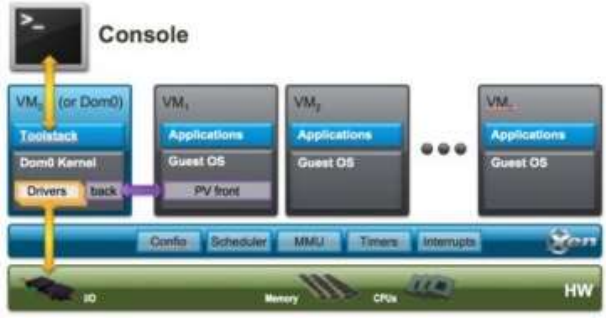

Slika 2 - Primer arhitekture Xen Hypervisor-a [2]

Tipovi particionisanja Xen Hypervisor-a su:

- $\quad$ soft

- hard

- $\quad$ particionisanje sa null scheduler-om.

U ovom radu je korišteno particionisanje sa null scheduler-om, ovaj tip particionisanja daje najbolje performanse i najmanji jitter. $U$ ovom načinu particionisanja svako virtuelno jezgro $(v C P U)$ je striktno mapirano jedan na jedan sa odgovarajućim $p C P U$. Ostali tipovi particionisanja se zasnivaju na raspoređivanju vCPU-a na pCPU-e na osnovu prioriteta što zahteva korišćenje određenog sistema za raspoređivanje (scheduler-a).

\section{BUILD XEN HYPERVISOR-A SA PETALINUX- OM}

PetaLinux alat poseduje sve neophodne stvari za prilagođavanje, build-ovanje i razvijanje embedded linux rešenja na Xilix sistemima. Prilagođen je da ubrza produktivnost dizajna, ovo rešenje i Xilinx alat za dizajniranje hardvera u mnogome olakšavaju razvoj Linux sistema za Zynq UltraScale+MPSoC, Zynq-7000 SoC i MicroBlaze. PetaLinux 2018.2 je korišten u ovom radu.

Zahtevi računara za instalaciju PetaLinux-a: 8GB RAM (preporučeni minimum za Xilinx alate), 2GHz CPU clock ili ekvivalentni (minimum 8 jezgara), 100GB slobodnog prostora na hard disku, operativni sistem: (Red Hat Enterprise Workstation/Server 7.2, 7.3, 7.4 (64-bit), CentOS 7.2, 7.3, 7.4 (64-bit) ili Ubuntu Linux 16.04.3 (64-bit) koji je izabran u ovom radu), root pristup nekim operacijama. PetaLinux alati trebaju biti instalirani kao non-root user, PetaLinux zahteva razne standardne razvojne alate i biblioteke koje moraju biti instalirane na Linux host-u. U tabeli 1 prikazan je tok razvoja Xen Hypervisor-a korišćenjem PetaLinux alata [6].

Tabela 1 - Tok dizajna [6]

\begin{tabular}{|l|l|}
\hline \multicolumn{1}{|c|}{$\begin{array}{c}\text { Koraci prilikom } \\
\text { dizajniranja }\end{array}$} & \multicolumn{1}{c|}{ Alat / Proces rada } \\
\hline $\begin{array}{l}\text { Pravljenje hardware } \\
\text { platforme }\end{array}$ & Vivado \\
\hline $\begin{array}{l}\text { Pravljenje PetaLinux } \\
\text { projekta }\end{array}$ & petalinux-create -t project \\
\hline $\begin{array}{l}\text { Inicijalizovanje PetaLinux } \\
\text { projekta }\end{array}$ & $\begin{array}{l}\text { petalinux-config --get-hw- } \\
\text { description }\end{array}$ \\
\hline $\begin{array}{l}\text { Konfigurisanje system- } \\
\text { level opcija }\end{array}$ & petalinux-config \\
\hline $\begin{array}{l}\text { Pravljenje korisničkih } \\
\text { komponenti }\end{array}$ & $\begin{array}{l}\text { petalinux-create -t } \\
\text { COMPONENT }\end{array}$ \\
\hline
\end{tabular}

\begin{tabular}{|l|l|}
\hline $\begin{array}{l}\text { Konfigurisanje Linux } \\
\text { kernel }\end{array}$ & petalinux-config -c kernel \\
\hline $\begin{array}{l}\text { Konfigurisanje Root } \\
\text { FileSystem-a }\end{array}$ & petalinux-config -c rootfs \\
\hline Build-ovanje sistema & petalinux-build \\
\hline Sistem deployment & petalinux-package \\
\hline Testiranje sistema & petalinux-boot \\
\hline
\end{tabular}

\section{PRIPREMA ZA BOOT-OVANJE PETALINUX IMAGE-A NA ULTRAZED PLOČI SA SD KARTICOM \\ Preduslovi: \\ - instalirano PetaLinux radno okruženje \\ - $\quad$ instaliran board support package $(B S P)$ \\ - $\quad$ serijska komunikacija je podešena sa baudrate- om 115200 \\ - UltraZed je podešen za boot-ovanje sa SD kartice, podesiti prekidače (boot mode switches SW2) na OFF-ON-OFF-ON}

\section{KORACI ZA BOOT-OVANJE PETALINUX-A NA HARDVER SA SD KARTICOM}

1. Ubaciti SD karticu u računar

2. Na karticu kopirati sledeće fajlove, ovi fajlovi se nalaze u direktorijumu <path_to_project>/images/linux:

$$
\begin{array}{ll}
\text { - } & \text { BOOT.bin } \\
\text { - } & \text { Image } \\
\text { - } & \text { system.dtb } \\
\text { - } & \text { xen.ub } \\
\text { - } & \text { rootfs.cpio.gz.u-boot }
\end{array}
$$

3. Povezati serjiski port ploče sa računarom

4. Otvoriti terminal na računaru (Putty, Kermit, minicom, gtkterm, itd.) i podesiti baud rate na 115200

5. Isključiti ploču

6. Postaviti boot mode preko prekidača na ploči kao što je opisano u prethodnoj sekciji

7. Ubaciti SD karticu u ploču

8. Uključiti ploču

9. Obratiti pažnju na terminal, pojaviće se boot poruke

Kada dom0 završi boot-ovanje može se pristupiti kreiranju jednostavnog domU guest-a. Potrebno je kopirati guest Image u dom0 filesystem, može se koristiti prebuilt PetaLinux Image kao domU guest. Svaki domU ukoliko se koristi bare-metal aplikacija, koja se kreira u Xilinx SDK random okruženju, mora imati .bin i .cfg fajlove. Fajl sa ekstenzijom .bin sadrži samu bare-metal aplikaciju i generiše se u Xilinx SDK random okruženju a .cfg fajl sadrži potreba podešava za Xen Hypervisor [4].

Primer .cfg fajla: 


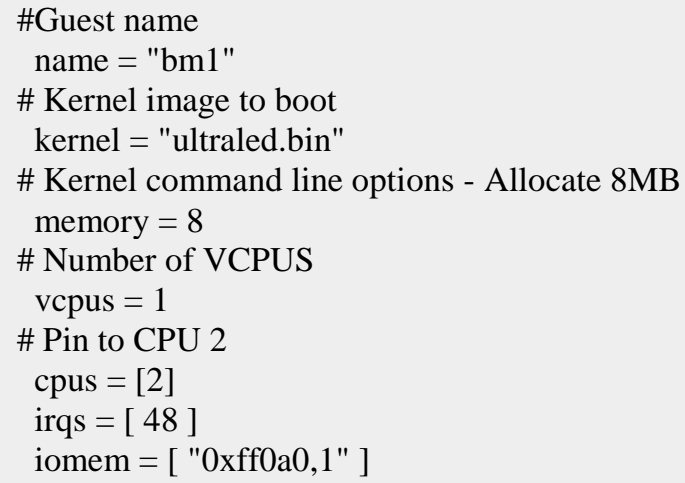

Da bi domU imao pristup na primer $U A R T-u$ i $G P I O$ ubačen je passthrough dodatkom u device-tree pre build-a [3]:

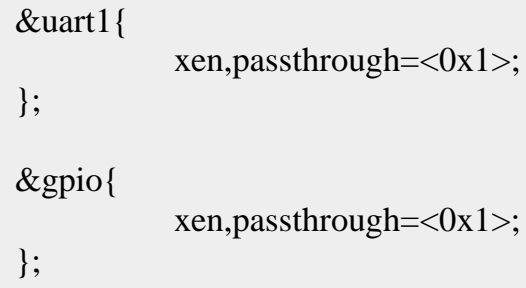

Nakon pozicioniranja u direktorijum sa .bin i .cfg fajlovima za kreiranje domU, koji će biti pokrenut samo na trećem jezgru, korištena je sledeća komanda:

$\$$ xl create -c example-simple.cfg gde je example-simple.cfg konfiguracioni fajl. Konzola guest-a se može napustiti sa Ctrl+]. U dom0 konzoli se mogu izlistati trenutni domU komandom: \$ xl list

Vraćanje u konzolu guest-a može se uraditi komandom: $\$ x l$ console <ime-guest-a>

A domU može biti isključen komandom:

$$
\text { \$ xl destroy <ime domU-a > }
$$

\section{7. “OVERHEAD” XEN HYPERVISOR-A}

Ovo poglavlje se bavi optimizacijom performansi Xen Hypervisor-a kao i upoređivanjem jitter-a bare-metal aplikacije "pod" Xen Hypervisor-om gde je CPU na kom je host tj. dom0 PetaLinux stresiran na $100 \%$ opretećenja $i$ bare-metal aplikacije koja se sama spušta na ploču bez Xen Hypervisor-a.

Xen Hypervisor podržava više različitih scheduler-a tj. sistema za rapoređivanje $v C P U$-ova. Posao hypervisor scheduler-a jeste da donosi odluke koji $v C P U$ od brojnih $v C P U$-ova različitih $V M$ će biti pokrenut na $p C P U$-ima i u koje vreme. Takođe je podržana opcija postojanja više različitih aktivnih scheduler-a u odvojenim grupama pCPU-a koji se nazivaju cpu-pool-ovi.

$C P U$-pool-ovi omogućavaju odvajanje fizičkih $C P U-a \mathrm{u}$ odvojene grupe pod nazivom cpu-pool. Svaki pool sadrži svoj potpuno odvojeni scheduler. Domain-i su dodeljeni pool-ovima pri kreiranju i mogu biti premeštani iz jednog pool-a u drugi.
Tipovi Xen Hypervisor scheduler-a [4]:

- Credit Scheduler - koji je opšte namene i podešen je po default-u

- $\quad$ Credit2 Scheduler - je naslednik Credit Scheduler-a, skalabilniji je i bolji je sa opterećenjima osetljivim na "latency" ali je pored toga baziran na kao scheduler opšte namene

- $\quad$ RTDS Scheduler - je real-time scheduler koji služi za podšku real-time opterećenja u cloud-u kao i za embedded i mobilnu virtuelizaciju

- ARINC653 Scheduler - je embedded (automobilska i avio industrija) real-time scheduler

- $\quad$ Null Scheduler - koji je korišten u ovom radu jer poseduje najbolje performanse jer nema scheduler-a tako da nema "donosenja odluka" i pomeranja $v C P U$-ova po $p C P U$-ima što dodatno smanjuje vreme, svaki $v C P U$ je dodeljen određenom $p C P U-u$.

$\mathrm{U}$ ovom radu bare-metal aplikacija je programirana da uključuje $\mathrm{i}$ iskljuluje tj. da trepće PS LED na UltraZed carrier card-u na osnovu čega se meri jitter.

Da bi se postigle najbolje performanse, najmanji overhead i najmanji interrupt latency ubačene su sledeće komande $u$ xen-overlay fajl kao xen-bootargs [5]:

$$
\begin{aligned}
& \text { sched=null } \\
& \text { vwfi=native }
\end{aligned}
$$

sched=null isključuje default Credit scheduler čime se dobija najniži overhead.

vwfi=native, gde $v w f i$ znači virtual wait for interrupt $\mathrm{tj}$. čekanje na virtuelni prekid, kada je postavljeno na „native“ smanjuje interrupt latency približno 60\% jer Hypervisor tada ne zadržava wfi i wfe komande koje su instrukcije ARM procesora za ,sleep“, podrazumevano tj. default podešavanje za vwfi je ,trap“. DEBUG opcija je takođe isključena, kada je uključena daje mnogo korisnih poruka i provera po ceni povećanog latency-a.

Kao što je prikazano na slici 4, jitter meren osciloskopom je $1,120 \mu$ s na UltraZed-EG ploči sa Xen Hypervisor-om.

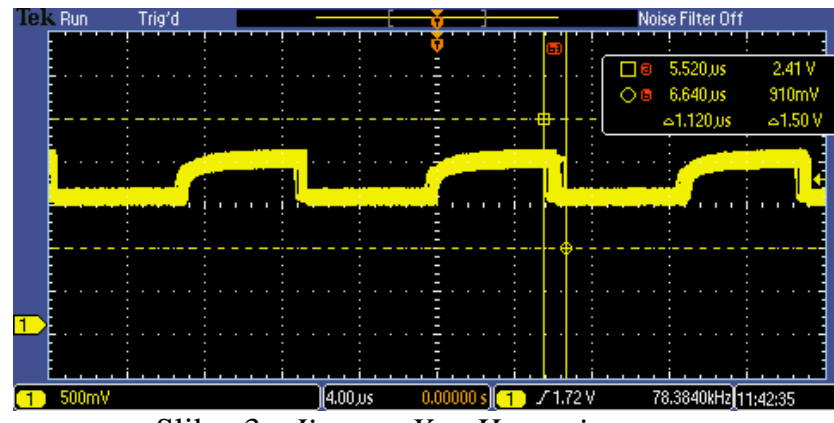

Slika 3 - Jitter sa Xen Hypervisor-om

Na slici 4 je prikazan jitter meren osciloskopom na ploči UltraZed-EG gde je bare-metal aplikacija za treptanje PS LED-a spuštena na ploču preko JTAG-a, naravno bez Xen Hypervisora, iz Xilnx SDK radnog okruženja. Jitter iznosi $800 \mathrm{~ns}$. 


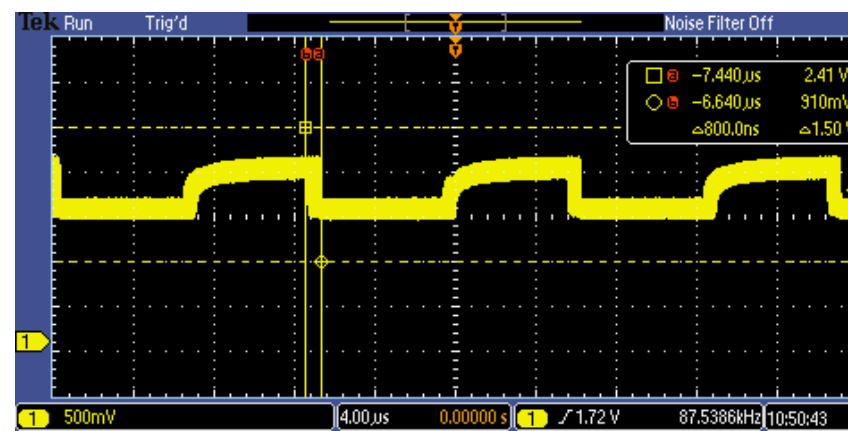

Slika 4 - Jitter bez Xen Hypervisor-a

Jitter ostaje isti prilikom stresiranja CPU-a na kom se nalazi dom0.

Izvršeno je testiranje sa aplikacijom koja pored uključivanja i isključivanja LED-a postavlja neke vrednosti $\mathrm{i}$ generiše tajmer interrupt svakih $10 \mu \mathrm{s}$. Izmeren je jitter od $3 \mu$ s što ukazuje da se Xen Hypervisor ima prevelik overhead da bi se koristio u sistemu za realtime simulacije, real-time signal processing, itd. Smanjenjem frekvencije interrupt-a na $15 \mu$ s jitter se smanjuje na vrednost od $1,120 \mu$ s što ukazuje na problem pri generisanju interrupt- $a$ visoke učestanosti. Na slici 5 prikazan je jitter pri frekvenciji interrupt-a od $10 \mu \mathrm{s}$.

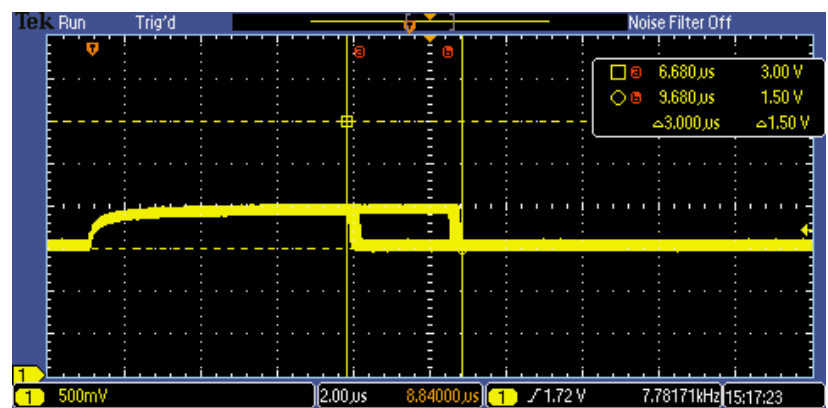

Slika 5 - Jitter sa aplikacijom koja genneriše interrupt-e

Takođe je izvršeno merenje korišćenjem iste aplikacije i default credit scheduler-a gde je dobijen jitter od $10 \mu \mathrm{s}$ pri frekvenciji interrupt- $a$ od $10 \mu$ s što je i prikazano na slici 6.

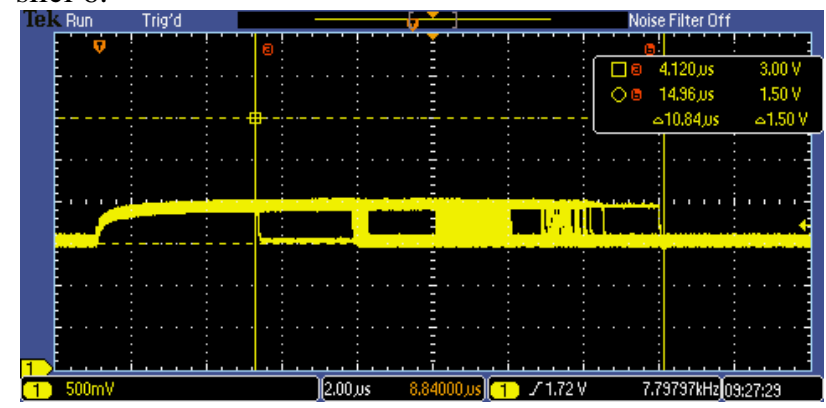

Slika 6 - Jitter sa credit scheduler-om i aplikacijom koja

\section{ZAKLJUČAK}

generiše interrupt-e

Dat je kratak pregled real-time particionisanja kao i njegova podela. Primećen je porast u potražnji real-time sistema sa sistemom za particionisanje. Objašnjen je overhead i jitter čije će se vrednosti smanjivati primenom određenih dodataka.
Dati su zahtevi računara za build-ovanje. Predstavljen je PetaLinux kao host tj. dom0 Xen Hypervisor-a. Objašnjen je proces build-ovanja korišćenjem PetaLinux-a kao i potrebna podešavanja za pokretanje sa SD kartice. Takođe opisuje pokretanje jednostavnog guest-a tj. domU-a i CPU pinning koji je veoma bitan za real-time particionisanje.

Izvršeno je upoređivanje jitter-a bare-metal aplikacije sa i bez Xen Hypervisor-a. Predstavljene su vrste sistema za raspoređivanje tj. vrste scheduler-a gde je ustanovljeno da null scheduler daje najbolje rezultate.

Izmeren je jitter na aplikaciji koja uključuje i isključuje LED velikom brzinom gde je otkriveno da je jitter Xen Hypervisor-a izmeren osciloskopom 1,120 $\mu \mathrm{s}$. Predstavljeni su rezultati merenja izvršavanja aplikacije koja pored uključivanja i isključivanja LED-a generiše tajmer interrupt svakih $10 \mu \mathrm{s}$. Zaključeno je da su najbolji rezultati dobijeni korišćenjem null scheduler-a $\mathrm{i}$ vwfi=native komande.

Nakon iznetih rezultata može se primetiti značajan overhead Xen Hypervisor-a pri konstantnom generisanju interrupt-a te se kao poboljšanje predlaže dodatna optimizacija performansi u slučaju interrupt-a koji se generiše na svakih 10 ili manje od $10 \mu$ s.

\section{LITERATURA}

[1] Donny Saveski. (2018, Sep.) zedboard.org. [Online] http://zedboard.org/sites/default/files/documentations/5264UG-AES-ZU3EGES-1-SOM-G-v1-1-V1.pdf

[2] Xilinx. (2018, Sep.) wiki.xilinx.com. [Online]. https://wiki.xen.org/wiki/Xen_Project_Software_Overview

[3] Xilinx. (2018, Sep.) wiki.xilinx.com. [Online]. http://www.wiki.xilinx.com/Building\%20Xen\%20Hypervis or\%20with\%20Petalinux\%202018.1

[4] xenproject.org. (2018, Sep.) wiki.xenproject.org. [Online]. https://wiki.xenproject.org/wiki/Xen_Project_Schedulers

[5] Stefano Stabellini. (2018, Sep.) xenproject.org. [Online]. blog.xenproject.org/author/stefano-stabellini/

[6] Xilinx. (2018, Sep.) xilinx.com. [Online]. https://www.xilinx.com/support/documentation/sw_manuals /xilinx2018_2/ug1144-petalinux-tools-referenceguide.pdf\#nameddest=PetaLinuxToolsInstallationSteps

\section{Kratka biografija:}

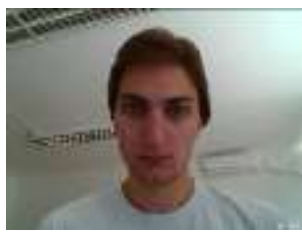

Milan Boberić rođen je u Zrenjaninu 1994. god. Master rad na Fakultetu tehničkih nauka iz oblasti

Mehatronike odbranio je 2018. god. kontakt: milanboberic94@gmail.com 